\title{
A general holographic metal/superconductor phase transition model
}

\author{
Yan Peng ${ }^{a}$ and Yunqi Liu ${ }^{b}$ \\ ${ }^{a}$ School of Mathematics and Computer Science, Shaanxi University of Technology, \\ Chaoyang road, Hanzhong, Shaanxi 723000, P.R. China \\ ${ }^{b}$ Department of Physics and Astronomy, Shanghai Jiao Tong University, \\ Dongchuan road, Shanghai 200240, P.R. China \\ E-mail: yanpengphy@163.com, liuyunqi@sjtu.edu.cn
}

ABSTRACT: We study the scalar condensation of a general holographic superconductor model in AdS black hole background away from the probe limit. We find the model parameters together with the scalar mass and backreaction can determine the order of phase transitions completely. In addition, we observe two types of discontinuities of the scalar operator in the case of first order phase transitions. We analyze in detail the effects of the scalar mass and backreaction on the formation of discontinuities and arrive at an approximate relation between the threshold model parameters. Furthermore, we obtain superconductor solutions corresponding to higher energy states and examine the stability of these superconductor solutions.

KEYwords: AdS-CFT Correspondence, Black Holes

ARXIV EPRINT: 1410.7234 


\section{Contents}

1 Introduction 1

2 A general model of superconductor in AdS black hole $\quad 2$

2.1 Equations of motion and boundary conditions 2

2.2 The scalar condensation in AdS black hole 4

$\begin{array}{ll}2.3 \text { The stability of various solutions } & 9\end{array}$

3 Conclusions

\section{Introduction}

The AdS/CFT correspondence has provided us a useful approach to describe strongly interacting systems holographically through weakly coupled gravitational duals [1-3]. One of the mostly studied gravity dual is the holographic superconductor, which is constructed by a scalar field coupled to a Maxwell field in an AdS black hole background [4-6]. It shows that the black hole becomes unstable and the scalar field condensates on the black hole background when the Hawking temperature of a black hole drops below a critical value. According to the holographic theory, this instability in the $(d+1)$ dimensional AdS black hole corresponds to a d dimensional metal/superconductor phase transition on the boundary. Generally speaking, this phase transition belongs to the second order. Since then a lot of holographic duals have been established in various gravity theories and the models turns out to be quite successful in giving the qualitative properties of superconductivity. Some other recent progress on holographic superconductors can be found in [7]-[26].

With a very complete holographic superconductor model in AdS black hole, it was announced in [27] that the scalar operator as a function of the temperature is always continuous. The instability of this gravity system corresponds to the second order phase transition. Lately, it was stated in $[28,29]$ that the holographic superconductor with the spontaneous breaking of a global U(1) symmetry via the Stückelberg mechanism allows the first order discontinuous phase transition to occur. Some further studies were carried out in $[30-32,49]$ by considering the matter fields' backreaction on the background. It was found in [32] that the light backreaction can trigger the first order phase transition but the heavy backreaction suppresses the first order phase transition in the AdS black hole background. Generally speaking, the phase transition is between normal state and superconducting state. Very recently a $\psi^{2}+\zeta \psi^{6}$ Stückelberg mechanism was discussed in the AdS soliton spacetime [50], which in particular admits new types of phase transitions between superconducting states. So it is very interesting to extend this new Stückelberg mechanism to the metal/superconductor system to explore the rich properties of holographic superconductors. 
The entanglement entropy is usually applied to study the degrees of freedom in strongly interacting systems when other methods might not be available. Ryu and Takayanagi [33, 34] have presented a simple and elegant way to calculate the entanglement entropy of a strongly interacting system from a gravity dual. The entanglement entropy has been applied to study the properties of phase transitions in various gravity theories and proved to be a good probe to give us new insights into the holographic superconductor models [35][45]. In the AdS black hole background, Albash and Johnson observed in [46] that the entanglement entropy as a function of temperature has a discontinuous slop at the transition temperature $T_{c}$ corresponding to the second order metal/superconductor phase transition. In contrast, there is a jump in the entanglement entropy when allowing the first order phase transition [46-49], which means that the entanglement entropy can be used to disclose the order of phase transitions. When considering a new Stückelberg model with a $\psi^{2}+\zeta \psi^{6}$ term in the AdS soliton background [50], the solutions in particular admits richer structures for the insulator/superconductor phase transition diagram. As a further step along this line, it is of great interest to generalize the investigation in [50] to AdS black hole and study general features of the metal/superconductor phase transitions through entanglement entropy approach in such a $\psi^{2}+\zeta \psi^{6}$ Stückelberg holographic superconductor model.

At zero temperature, it was found in [51] that different energy states appear for the scalar condensation in the AdS black hole background. Superconductor solutions corresponding to different states were also mentioned with the behaviors of the scalar fields away from the zero temperature limit [52]. When applying the discussion to holographic insulator/superconductor system, different states will turn out by increasing the critical chemical potential from zero to critical values with other parameters fixed [53]. It concluded in [53] that the second and third states are less stable due to the oscillations of scalar field in the radial direction and the first state is related to the superconductor solutions in the AdS soliton spacetime [54]. It is interesting to extend the discussion to examine whether there are different states in more general holographic superconductor models and also further explore the stability of various possible states.

The next section is organized as follows. In part A, we introduce a Stückelberg holographic model in the four dimensional AdS black hole background. And in part B, we discuss in detail the scalar condensation and the holographic entanglement entropy of the system in superconductor phase. Part C is devoted to the stability of various solutions with different energy states. We summarize and discuss our main results in section III.

\section{A general model of superconductor in AdS black hole}

\subsection{Equations of motion and boundary conditions}

We begin with a generalized Stückelberg Lagrange density containing a Maxwell field and a scalar field,

$$
\mathcal{L}=R+\frac{6}{L^{2}}-\gamma\left[\frac{1}{4} F^{\mu \nu} F_{\mu \nu}+(\partial \psi)^{2}+m^{2}|\psi|^{2}+G(\psi)(\partial p-A)^{2}\right]
$$


where $A_{\mu}$ and $\psi$ are the Maxwell field and a charged scalar field with mass $m^{2}$, respectively. $-3 / L^{2}$ is the negative cosmological constant, where $L$ is the AdS radius which will be scaled unity in our calculation. $\gamma$ is the backreaction parameter describing the effects of matter fields on the background. When $\gamma \rightarrow 0$, the backreaction of the matter fields on the background becomes negligible and the metric solutions reduce to the pure AdS black hole spacetime. We will establish a general Stückelberg holographic model in AdS black hole by considering a simple form $G(\psi)=\psi^{2}+\zeta \psi^{n}$ with $n \in N$, where $\zeta$ is the model parameter. With the gauge symmetries $A \rightarrow A+\partial \Lambda$ and $p \rightarrow p+\Lambda$, we fix $p=0$ in the following discussion.

The metric and other fields of interest are parameterized as follows:

$$
\begin{aligned}
d s^{2} & =-g(r) e^{-\chi(r)} d t^{2}+\frac{d r^{2}}{g(r)}+r^{2}\left(d x^{2}+d y^{2}\right) \\
A & =\phi(r) d t \\
\psi & =\psi(r) .
\end{aligned}
$$

With this ansatz, the Hawking temperature of the black hole is given by

$$
T_{H}=\frac{g^{\prime}\left(r_{+}\right) e^{-\chi\left(r_{+}\right) / 2}}{4 \pi}
$$

where $r_{+}$corresponds to the horizon of the black hole satisfying $g\left(r_{+}\right)=0$.

We can obtain equations of motion from the action

$$
\begin{aligned}
\chi^{\prime}+\gamma\left[r \psi^{\prime 2}+\frac{r}{g^{2}} e^{\chi} \phi^{2} G(\psi)\right] & =0, \\
g^{\prime}-\left(\frac{3 r}{L^{2}}-\frac{g}{r}\right)+\gamma r g\left[\frac{1}{2} \psi^{\prime 2}+\frac{1}{4 g} e^{\chi} \phi^{\prime 2}+\frac{m^{2}}{2 g} \psi^{2}+\frac{1}{2 g^{2}} e^{\chi} \phi^{2} G(\psi)\right] & =0, \\
\phi^{\prime \prime}+\left(\frac{2}{r}+\frac{\chi^{\prime}}{2}\right) \phi^{\prime}-\frac{2 G(\psi)}{g} \phi & =0, \\
\psi^{\prime \prime}+\left(\frac{2}{r}-\frac{\chi^{\prime}}{2}+\frac{g^{\prime}}{g}\right) \psi^{\prime}-\frac{m^{2}}{g} \psi+\frac{1}{2 g^{2}} e^{\chi} \phi^{2} G^{\prime}(\psi) & =0,
\end{aligned}
$$

where $G^{\prime}(\psi)$ represents the derivative of $\psi$. Since the equations are coupled and nonlinear, we have to solve these equations by numerically integrating them from the horizon out to the infinity.

By considering behaviors of solutions at the horizon $r_{+}$, we find that there are four independent parameters $r_{+}, \psi\left(r_{+}\right), \phi^{\prime}\left(r_{+}\right)$and $\chi\left(r_{+}\right)$at the horizon. The scaling symmetry

$$
r \rightarrow a r, \quad t \rightarrow a t, \quad \phi \rightarrow a \phi, \quad g \rightarrow a^{2} g
$$

can be used to set $r_{+}=1$. These equations are also invariant under another scaling,

$$
e^{-\chi} \rightarrow b^{2} e^{-\chi}, \quad t \rightarrow b t, \quad \phi \rightarrow \phi / b,
$$

which enable us to choose an arbitrary value of $\chi\left(r_{+}\right)$. With this eq. (2.9), we set $\chi(r \rightarrow$ $\infty)=0$ to recover the AdS boundary. 
Near the asymptotic AdS boundary $(r \rightarrow \infty)$, the asymptotic behaviors of the scalar and Maxwell fields are

$$
\psi=\frac{\psi_{-}}{r^{\lambda_{-}}}+\frac{\psi_{+}}{r^{\lambda_{+}}}+\cdots, \phi=\mu-\frac{\rho}{r}+\cdots,
$$

with $\lambda_{ \pm}=\left(3 \pm \sqrt{9+4 m^{2}}\right) / 2$, where $\mu$ and $\rho$ can be interpreted as the chemical potential and charge density in the dual theory respectively. When $\psi(r)=0$, we get the analytic solutions in normal phase, a Reissner-Nordstrom-AdS black hole, which is given by

$$
g=\frac{r^{2}}{L^{2}}-\frac{2 M}{r}+\frac{\gamma \rho^{2}}{4 r^{2}}, \quad \chi=0, \quad \phi=\rho\left(\frac{1}{r_{+}}-\frac{1}{r}\right),
$$

where $\mathrm{M}$ is the integration constant that can be interpreted as the mass of the black hole. It is known that the black hole is unstable when the temperature $\mathrm{T}$ is smaller than a critical temperature $T_{c}$. For low temperature $T<T_{c}$, a hairy black hole with $\psi(\infty)=0$ appears. To get the hairy black hole, we will fix $\psi_{-}=0$ and the phase transition in the dual CFT is described by the operator $\psi_{+}=<O_{+}>$in the following discussion. For $m^{2}>-\frac{9}{4}$ above the $\mathrm{BF}$ bound [55], the second scalar operator $\psi_{+}$is always normalizable. For each fixed value $\psi\left(r_{+}\right)$at the horizon, we take $\phi^{\prime}\left(r_{+}\right)$as the shooting parameter to search for the solutions satisfying the boundary conditions $\psi_{-}=0$.

\subsection{The scalar condensation in AdS black hole}

It was stated that a $\psi^{2}+\zeta \psi^{6}$ Stückelberg mechanism brings richer physics in the holographic insulator/superconductor phase transitions in the AdS soliton spacetime [50]. In the holographic metal/superconductor phase transition, we find the results are qualitative similar when $n \geqslant 5$. So we will focus on the case of $n=6$ in this paper similar to the discussions in ref. [50] for simplicity. We start with studying the free energy of the system. The free energy of the field theory is determined by the integral of the lagrange density eq. (1) evaluated on-shell or $F=-T \widetilde{S}$, where $\widetilde{S}=\frac{1}{V} \int L d V$. However, this integration will in general be divergent and suitable counterterms have to be added [12, 23]. The interesting quantity is the difference in free energy between the superconductor and normal phases.

$$
\Delta F=F_{\text {superconductor }}-F_{\text {normal }} .
$$

We show the free energy as a function of temperature in figure 1 with $\gamma=0.1, m^{2}=-2$ and various $\zeta$. It can be seen from $(a),(b)$ and $(c)$ in figure 1 that, for the small model parameters $(\zeta=0,0.2$ or 0.3$), \Delta F$ decreases smoothly near the critical temperature $T_{c}$ indicating the second order phase transitions from normal state into superconducting state. What's more, in $(c)$ the plot with $\zeta=0.3$, besides the second order phase transition at the critical temperature $T_{c}$, the free energy develops a "swallow tail" at $T \approx 0.0506$, a typical signal for a first order phase transition, indicating that there is a new phase transition within the superconducting phase. ( $d$ ) of figure 1 shows that as $\zeta$ increases to $0.6, \Delta F$ develops a discontinuity in the first derivative of the free energy with respect to temperature at critical temperature $T_{c}$, which implies that a strong St $\ddot{u}$ ckelberg mechanism with $\zeta=0.6$ triggers the first order phase transition. 

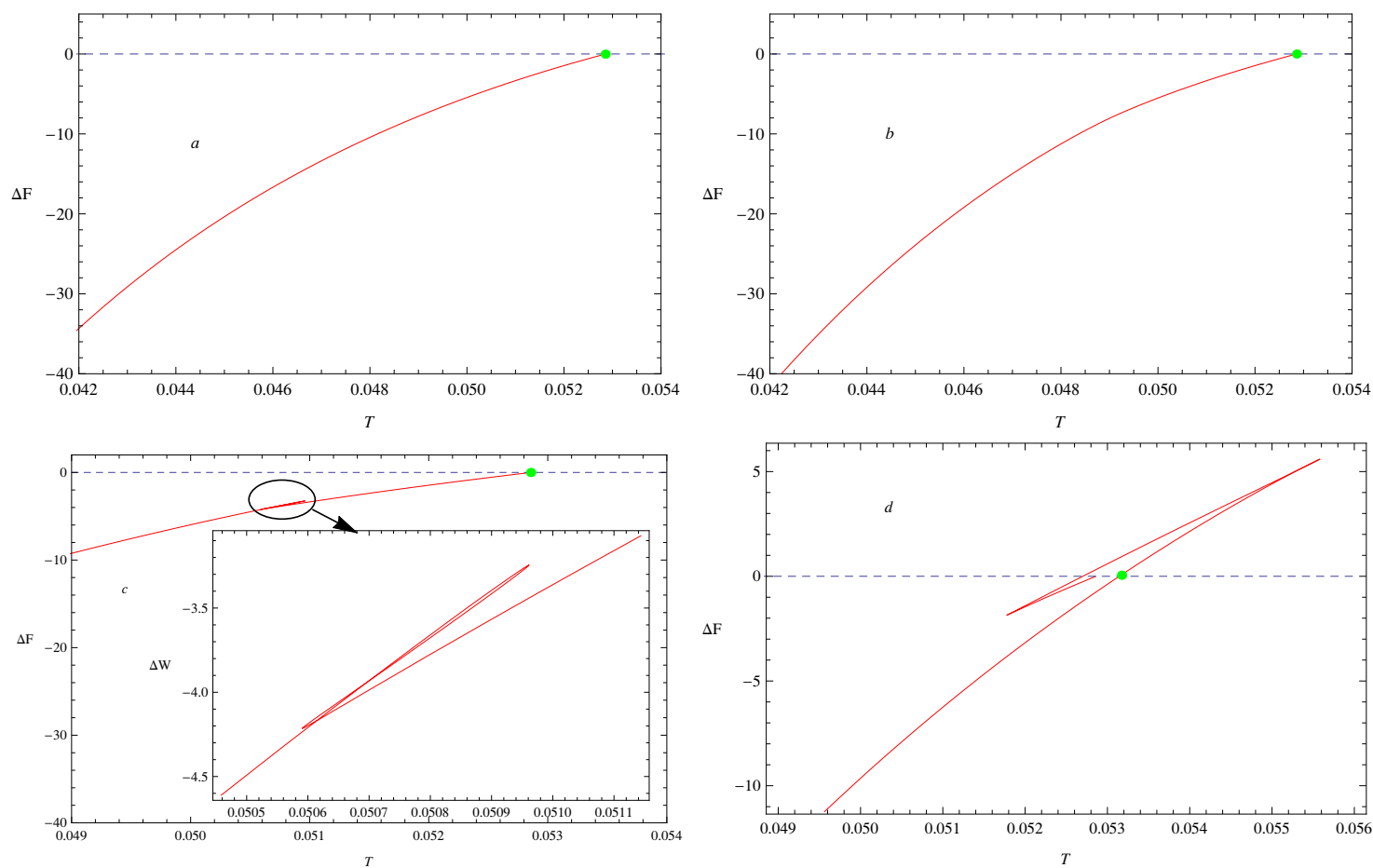

Figure 1. (Color online) The free energy as a function of $T$ with $\mu=1, m^{2}=-2, \gamma=0.1$ and various $\zeta$ : $(a)$ the case $\zeta=0,(b)$ the case $\zeta=0.2,(c)$ the case $\zeta=0.3,(d)$ the case $\zeta=0.6$. The blue dashed line in each panel corresponds to the free energy of a black hole in normal state. And the green solid points in each panel indicate the critical phase transition point $T_{c}$ between normal and superconductor phase.

We could also detect the properties of phase transitions by studying the condensation of the scalar operator for different values of $\zeta$. In figure 2 , we can see that the operator $\left\langle O_{+}\right\rangle^{1 / \lambda_{+}}$is monotonically as a function of temperature in $(a)$ with $\zeta=0$ and $(b)$ with $\zeta=$ 0.2 around the phase transition point. However, in $(c)$ as we choose the parameter $\zeta=0.3$, the curve starts from $\left\langle O_{+}\right\rangle^{1 / \lambda_{+}}=0$ at the critical temperature $T_{c}$ signaling a second order phase transition, and a dump of the scalar operator appears at $T \approx 0.0506$ corresponding to the first order discontinuity in $(c)$ of figure 1 . In cases that the transition occurs at $T_{c}$, the normal phase transfers into superconducting phase as we decrease the temperature. When the phase transition is second order, we have checked the mean field exponents and find the condensate approaches zero as $\left\langle O_{+}>\propto\left(T_{c}-T\right)^{1 / 2}\right.$. This is reasonable since $G(\psi)=\psi^{2}+\zeta \psi^{6} \approx \psi^{2}$ (or independent of $\zeta$ ) around the phase transition point $T_{c}$. For example, the curves in (a), (b) and (c) of figure 2 correspond to $\left\langle O_{+}\right\rangle \approx 2.4\left(T_{c}-T\right)^{1 / 2}$ around $T_{c}$. As the model parameter $\zeta$ grows up, it can be seen in $(\mathrm{d})$ of figure 2 . that the operator $\left\langle O_{+}\right\rangle^{1 / \lambda_{+}}$starts from a finite value 0.49 at $T_{c}$, which means the transition from the normal phase to the superconductor belongs to the first order phase transition.

For the rich phases structure in the boundary theory in figure 1, in the gravity side we can use the gravity solotions to characterize the difference between these phases. In figure 3, fixing the coordinate $r=r_{0}=5$ (outside the horizon or $r_{0} \geqslant r_{+}$), we depict 

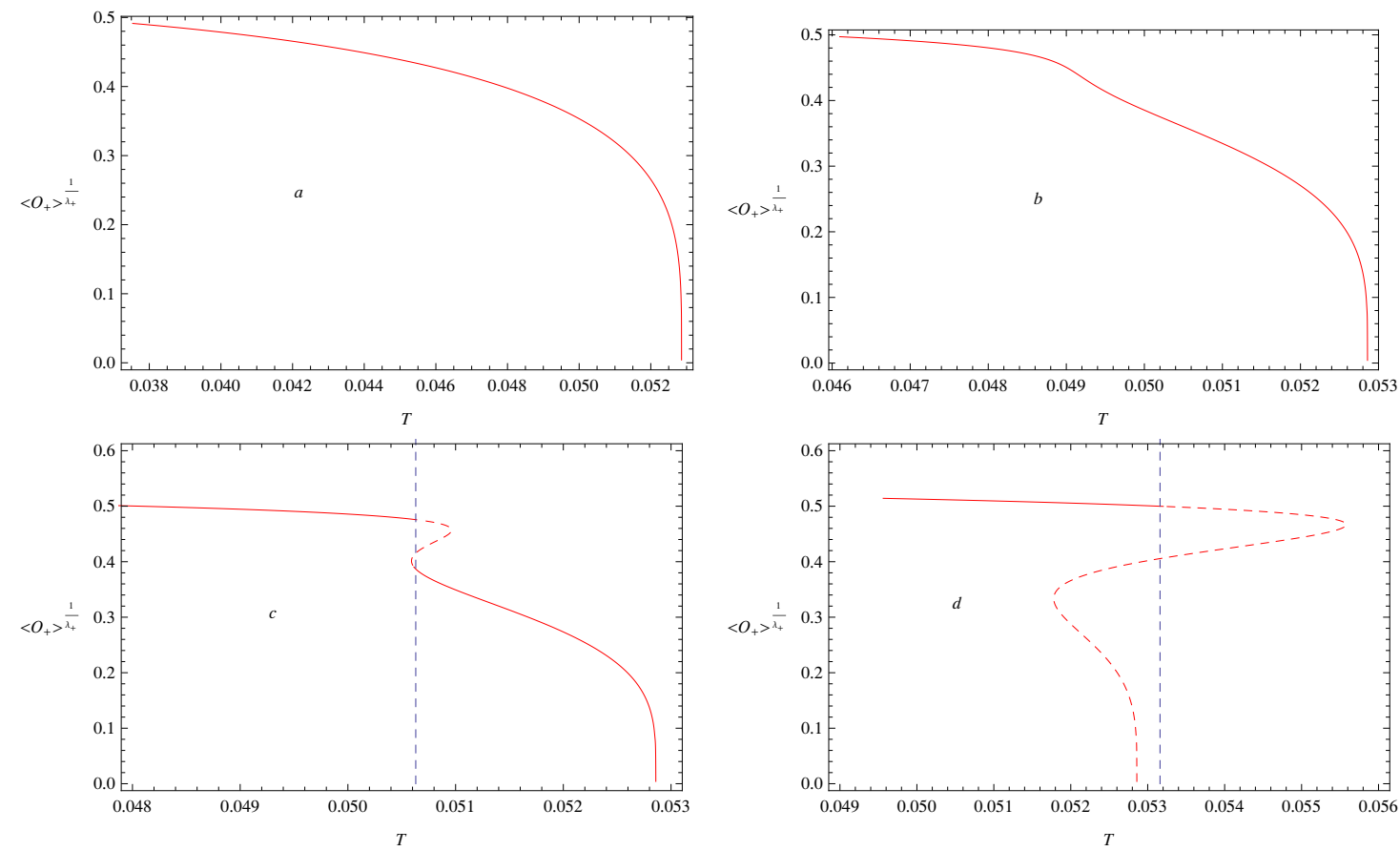

Figure 2. (Color online) The scalar operator as a function of $T$. We take $\mu=1, m^{2}=-2, \gamma=0.1$ and $\zeta$ varies as: $(a)$ the case $\zeta=0,(b)$ the case $\zeta=0.2,(c)$ the case $\zeta=0.3,(d)$ the case $\zeta=0.6$. The red solid line in each panel corresponds to the superconductor phase.

the metric $g\left(r_{0}\right)$ as a function of temperature T. In (a), (b) and (c) of figure 3, we find $g\left(r_{0}\right)$ has a discontinuous slop with respect to $\mathrm{T}$ at the temperature $T=0.05286$, which corresponding to the second order phase transition points in (a), (b) and (c) of figure 1. Further, in the first order phase transition points in (c) and (d) between superconducting phases in figure 1, we find jumps of $g\left(r_{0}\right)$. Then we concluded that the discontinuity in the first derivative of $g\left(r_{0}\right)$ corresponds to the second order phase transition and the jump of $g\left(r_{0}\right)$ corresponds to the first order phase transition.

In the following discussion, we pay our attention to the holographic entanglement entropy(HEE) of the phase transition system. The authors in refs. [33, 34] have presented a proposal to compute the entanglement entropy of conformal field theories (CFTs) from the minimal area surface in gravity side. We consider a subsystem $\tilde{A}$ with a straight strip geometry described by $-\frac{l}{2} \leqslant x \leqslant \frac{l}{2}, 0 \leq y \leq \tilde{L}$, where $l$ is defined as the size of region $\tilde{A}$, and $\tilde{L}$ is a regulator which is set to be infinity. Minimizing the area of hypersurface $\gamma_{\tilde{A}}$ whose boundary is the same as the stripe $\tilde{A}$, the entanglement entropy for a belt geometry can be expressed as [46]

$$
S=\int_{\varepsilon}^{z_{*}} d z \frac{z_{*}^{2}}{z^{2}} \frac{1}{\sqrt{\left(z_{*}^{4}-z^{4}\right) z^{2} g(z)}}-\frac{1}{\varepsilon}
$$

with

$$
\frac{l}{2}=\int_{\varepsilon}^{z_{*}} d z \frac{z^{2}}{\sqrt{\left(z_{*}^{4}-z^{4}\right) z^{2} g(z)}}
$$



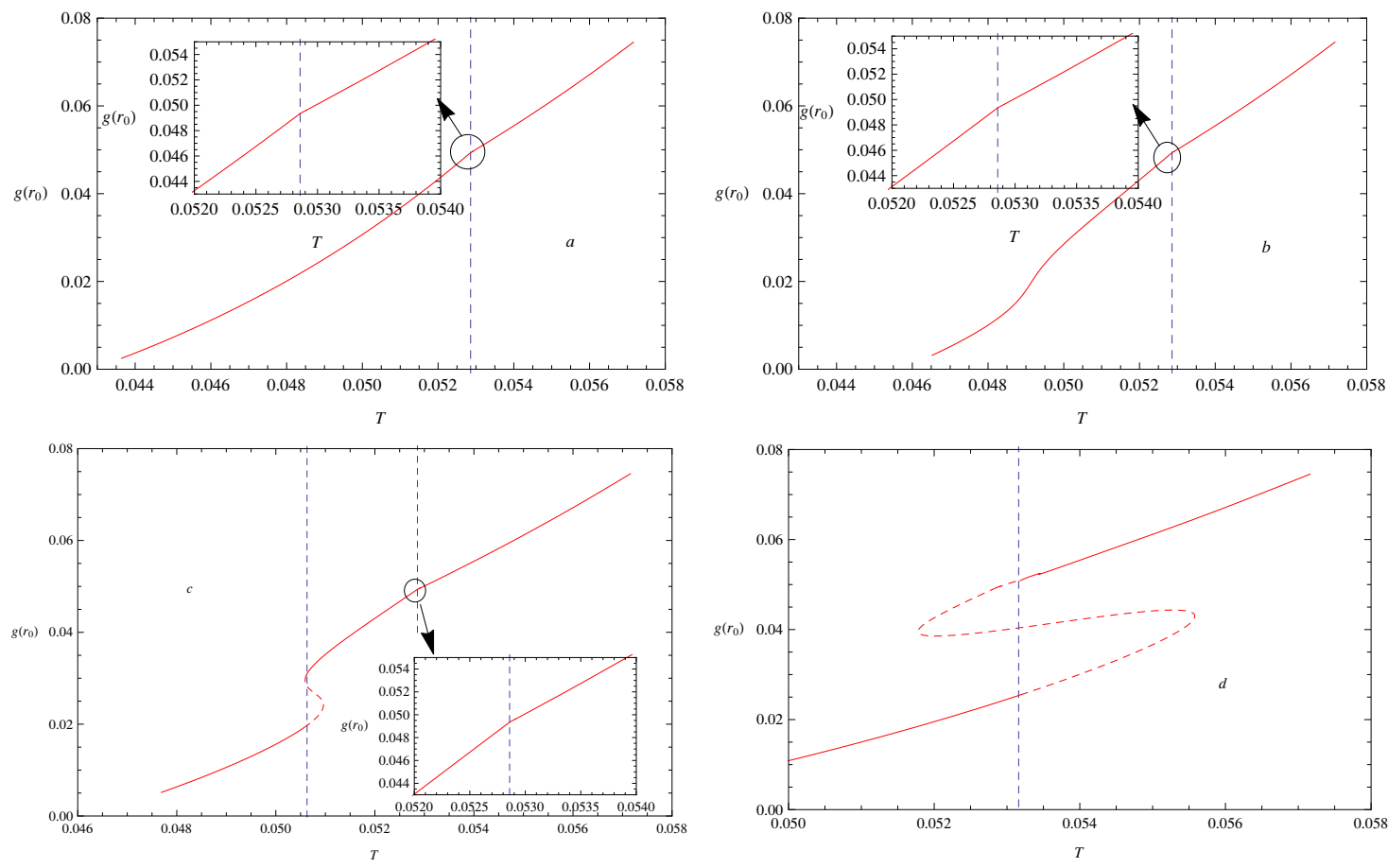

Figure 3. (Color online) The metric solution $g\left(r_{0}\right)$ as a function of $T$ with $r_{0}=5, \mu=1$, $m^{2}=-2, \gamma=0.1$ and various $\zeta:(a)$ the case $\zeta=0,(b)$ the case $\zeta=0.2,(c)$ the case $\zeta=0.3,(d)$ the case $\zeta=0.6$.

where $z_{*}$ satisfies the condition $\left.\frac{d z}{d x}\right|_{z_{*}}=0$ with $z=\frac{1}{r}$ and the UV cutoff $r=\frac{1}{\varepsilon}$ has been taken into consideration.

Now we exhibit properties of the phase transition through behaviors of the entanglement entropy. We plot the entanglement entropy as a function of the temperature $T$ in figure 4 with $\mu=1, \gamma=0.1$ and various $\zeta$. The blue dashed lines describe the HEE of the normal phase as a function of temperature, while the red solid lines correspond to the HEE of the superconducting phase. In $(a)$ with $\zeta=0$ and $(b)$ with $\zeta=0.2$ of figure 4 , the holographic entanglement entropy decreases continuously as temperature decreases, and there are discontinuous slops at the transition temperature $T_{c}$, which imply the phase transitions at the critical temperature $T_{c}$ are second order. In $(c)$ with $\zeta=0.3$, the entanglement entropy continuously decreases at phase transition point $T_{c}$ indicating a second order phase transition, and it also has a jump around $T=0.0506$ which corresponds to the "swallow tail" in free energy $\Delta F$ in $(c)$ of figure 1 and the dump in the order operator in $(c)$ of figure 2, which implies a first order phase transition in the superconducting phase. When we set the parameter $\zeta=0.6$ in $(d)$, we can see that at the critical temperature $T_{c}$ the entanglement entropy develops a discontinuous jump implying a first order phase transition. From above discussion, we note that the Stückelberg mechanism provides richer physics in metal/superconductor phase transitions. The entanglement entropy can be used to distinguish the order of phase transitions in our general holographic superconductor model. 

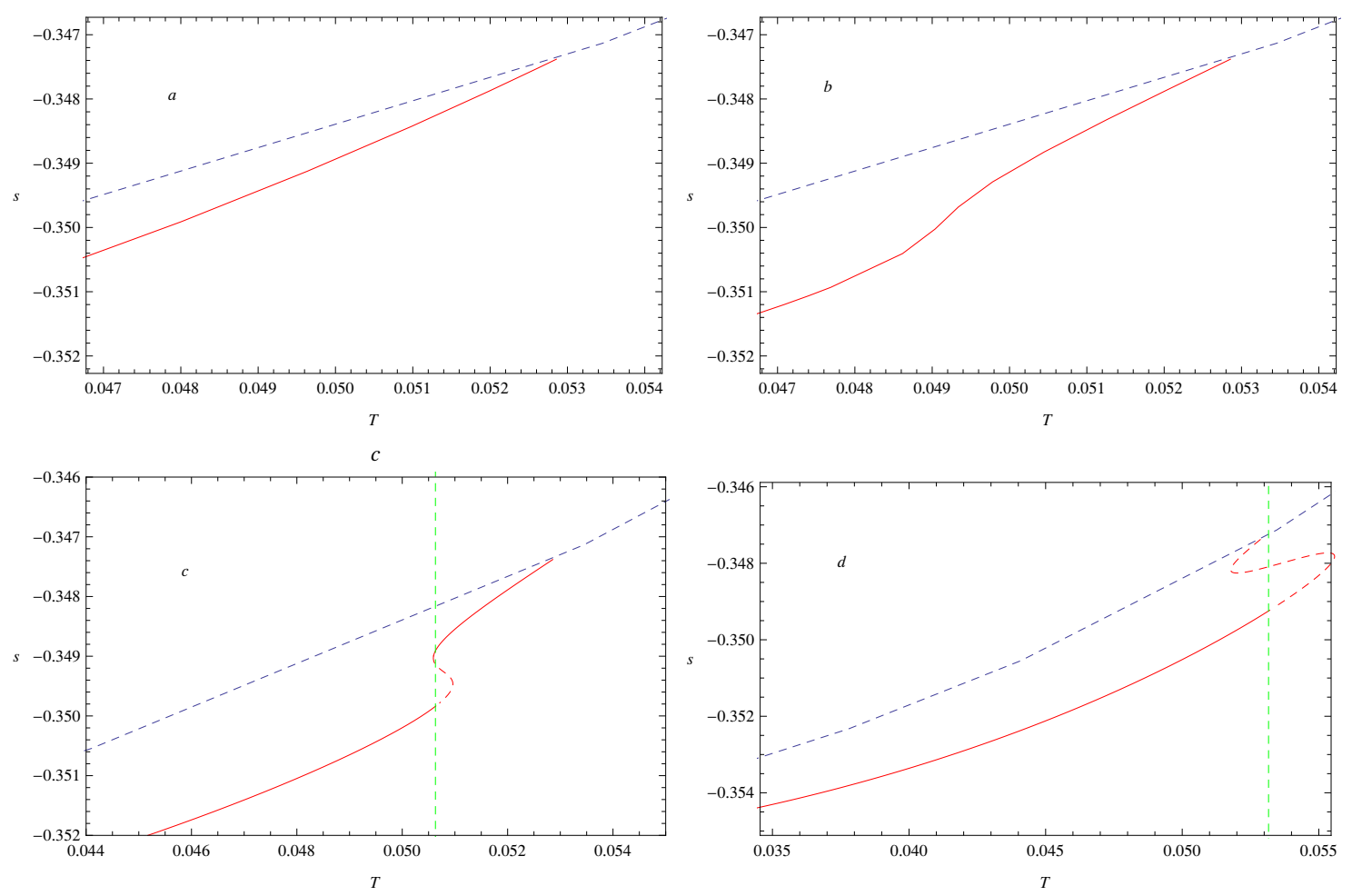

Figure 4. (Color online) The entanglement entropy as a function of the temperature $T$ for $\mu=1$ and $l=2$. The blue dashed line in each panel corresponds to the entanglement entropy of a pure AdS black hole. The red solid curves show the entanglement entropy of the superconductor phase. The cases are: (a) $\zeta=0, m^{2}=-2, \gamma=0.1$, (b) $\zeta=0.2, m^{2}=-2, \gamma=0.1$, (c) $\zeta=0.3, m^{2}=-2$, $\gamma=0.1$ and $(\mathrm{d}) \zeta=0.6, m^{2}=-2, \gamma=0.1$

According to the above discussions, we conclude that the Stückelberg mechanism triggers the second order phase transition at $T_{c}$ for small $\zeta$. When we choose a larger $\zeta$, an additional first order phase transition may appear in the superconducting phase, whereas the phase transition at $T_{c}$ still corresponds to the second order. However, there is only the first order phase transition at $T_{c}$ for very large $\zeta$. We define two parameters $\bar{\zeta}$ and $\widetilde{\zeta}$ as threshold values, for $\zeta \leqslant \tilde{\zeta}$ the second order phase transition appears at the critical temperature $T_{c}$. And for $\tilde{\zeta}<\zeta<\bar{\zeta}$, the system experiences a second order phase transition at $T_{c}$ and an additional first order transition in the superconducting phase. When $\zeta \geqslant \bar{\zeta}$, there is only the first order phase transition at the critical temperature. Table 1 shows the values of $\tilde{\zeta}$ and $\bar{\zeta}$ for various $m^{2}$ and $\gamma$. From the table, we obtain an approximate relation $\bar{\zeta} \approx 2 \tilde{\zeta}$. In order to see the effects of the backreaction $\gamma$ and mass $m^{2}$ on the condensation more clearly, we show $\tilde{\zeta}$ and $\bar{\zeta}$ as a function of $m^{2}$ with $\gamma=0.1$ in the left panel of figure 5 and reach the conclusion that a less negative mass makes the first order phase transition easier to happen (or smaller threshold parameters $\tilde{\zeta}$ and $\bar{\zeta}$ ). With a fixed $m^{2}$ and various $\gamma$, the right panel of figure 5 signals that strong backreaction promote the appearance of first order phase transitions. From the pictures, we also mention that the relation $\bar{\zeta} \approx 2 \tilde{\zeta}$ holds very well. 

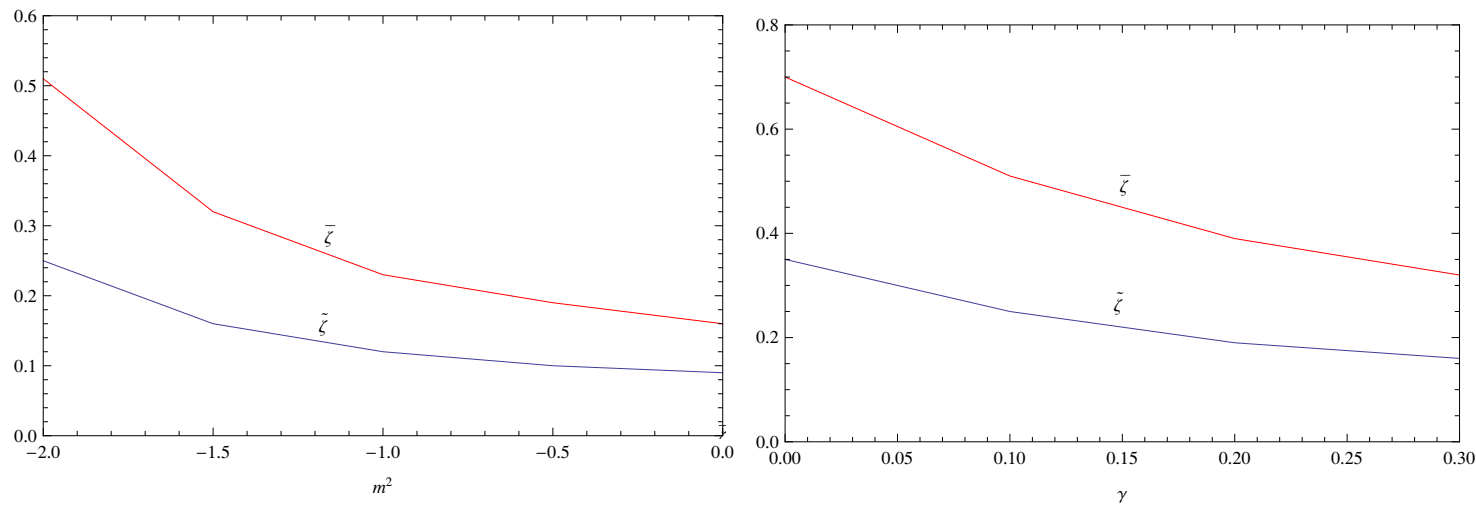

Figure 5. (Color online) The behaviors of the threshold values of $\bar{\zeta}$ (red) and $\widetilde{\zeta}$ (blue). The left panel corresponds to the case of $\gamma=0.1$ and various $m^{2}$. The right one shows the case $m^{2}=-2$ and various $\gamma$.

\begin{tabular}{|cccc|}
\hline & $\gamma=0.1, m^{2}=-2$ & $\gamma=0.2, m^{2}=-2$ & $\gamma=0.1, m^{2}=-1$ \\
\hline$\widetilde{\zeta}$ & 0.25 & 0.19 & 0.12 \\
$\bar{\zeta}$ & 0.51 & 0.39 & 0.23 \\
\hline
\end{tabular}

Table 1. The values of $\tilde{\zeta}$ and $\bar{\zeta}$ for various $m^{2}$ and $\gamma$.

We would like to give a glance at the effects of the scalar mass on the phase transition from the equations of motion. Let us rewrite eq. (7) into the following form:

$$
\psi^{\prime \prime}+\left(\frac{2}{r}-\frac{\chi^{\prime}}{2}+\frac{g^{\prime}}{g}\right) \psi^{\prime}-\left[m^{2}-\frac{1}{g} e^{\chi} \phi^{2}\left(1+3 \zeta \psi^{4}\right)\right] \frac{\psi}{g}=0 .
$$

We consider the last term in the left side as the effective mass $m_{\text {eff }}^{2}(\psi)=m^{2}-$ $\frac{1}{g} e^{\chi} \phi^{2}\left(1+3 \zeta \psi^{4}\right)$. For $\zeta=0$, it returns to the model without Stückelberg mechanism. For $\zeta$ above the threshold parameters, this term deforms the scalar field to prompt the appearance of first order phase transition. When we choose a more negative scalar mass $m^{2}$, the effective mass is mostly dominated by the scalar mass. For a less negative mass $m^{2} \approx 0$, the parameter $\zeta$ will play a dominant role in the scalar condensation and we need a smaller parameters $\tilde{\zeta}$ and $\bar{\zeta}$ to trigger the first order phase transitions.

\subsection{The stability of various solutions}

For each value $\psi\left(r_{+}\right)$, we find discrete values $\phi^{\prime}\left(r_{+}\right)$satisfying the boundary conditions $\psi_{-}=0$. As we choose various $\psi\left(r_{+}\right)$, we obtained different families of solutions for $\psi(r)$ that satisfying the asymptotic boundary condition. The solutions can be labeled by the number of times that $\psi(r)$ vanishes. The plots in figure 6 show the scalar fields corresponding to the first three states with $\gamma=0.1, m^{2}=-2, \zeta=0$ and $\psi\left(r_{+}\right)=0.1$. The scalar field of the first state in figure 6 starts from $\psi(1)=0.1$ at the horizon and decreases monotonically to zero as 


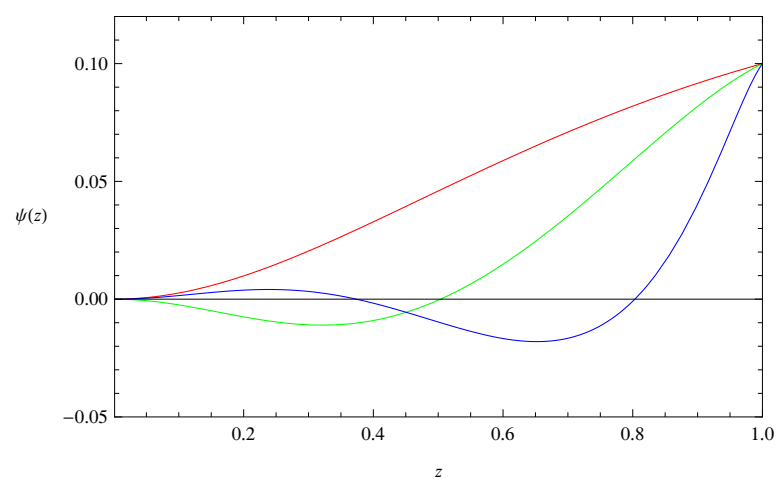

Figure 6. (Color online) The behaviors of the scalar fields $\psi(z)$ with $z=\frac{1}{r}$ and $\psi(1)=0.1$. The three curves correspond to the solutions: the 1st state $\phi^{\prime}(1)=3.929$ (Red), the 2nd state $\phi^{\prime}(1)=7.809$ (Green) and the 3rd state $\phi^{\prime}(1)=9.916$ (Blue) with $\gamma=0.1, m^{2}=-2$ and $\zeta=0$.
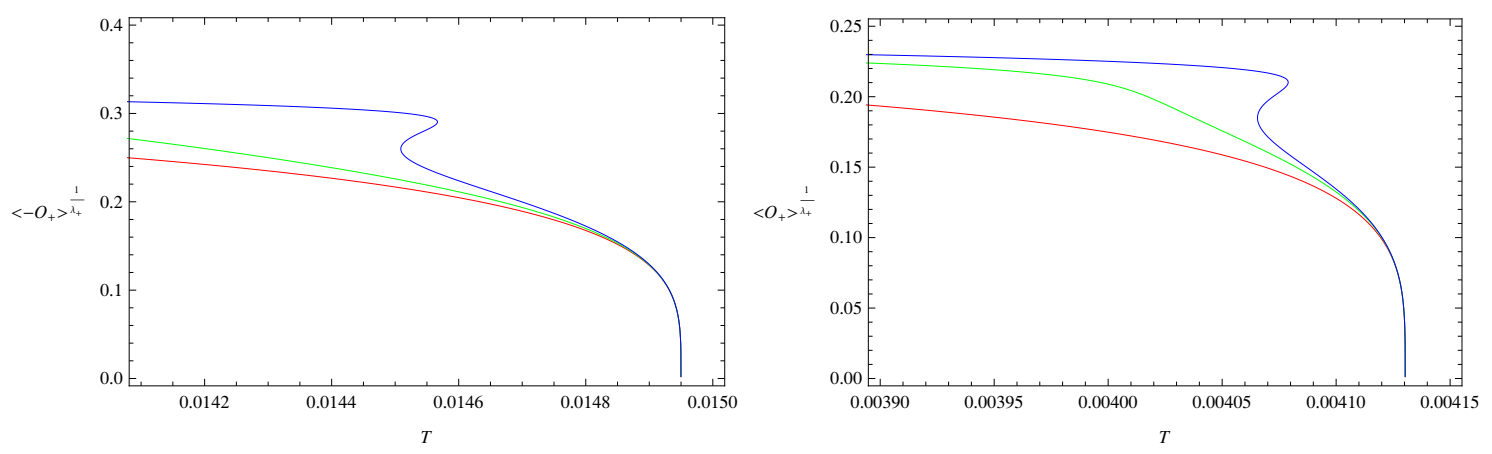

Figure 7. (Color online) The condensation of the scalar operators. The left panel corresponds to the case of $\gamma=0.1, m^{2}=-2, T_{c}=0.0149$ and $\zeta$ varies as $\zeta=0(\operatorname{Red}), \zeta=0.01$ (Green), $\zeta=0.02$ (Blue). The right one shows the case $\gamma=0.1, m^{2}=-2, T_{c}=0.0041$ and $\zeta$ varies as $\zeta=0$ (Red), $\zeta=0.003$ (Green), $\zeta=0.004$ (Blue).

approaching the boundary. And the higher states of the green and blue curves correspond to scalar fields with oscillations, which are similar to the case in AdS soliton background [53].

Now we turn to study behaviors of the scalar operator with $\gamma=0.1, m^{2}=-2$ and various model parameters $\zeta$ in figure 7 . We find the Stückelberg mechanism can trigger first order discontinuities in scalar condensation of all states. For the second state in the left panel of figure 7 is with $0.01<\tilde{\zeta}<0.02$ and the third state in the right panel of figure 7 , $0.003<\tilde{\zeta}<0.004$. Compared with the results $\widetilde{\zeta}=0.25$ of the first state in table 1 , we find the solutions with a lower state corresponds to a larger threshold model parameter $\widetilde{\zeta}$, above which first order phase transitions will appear in the superconducting phase. That is to say the superconductor solutions with higher states changes dramaticly with a small perturbation of the model parameter $\zeta$.

In order to study the stability of the states, we show the free energy of the systems corresponding to different states with $\gamma=0.1, m^{2}=-2$ and $\zeta=0$ in figure 8 . We find that the higher state corresponds to larger free energy. So we can label superconductor solutions as the first, the second and the third energy states through various increasing grand 


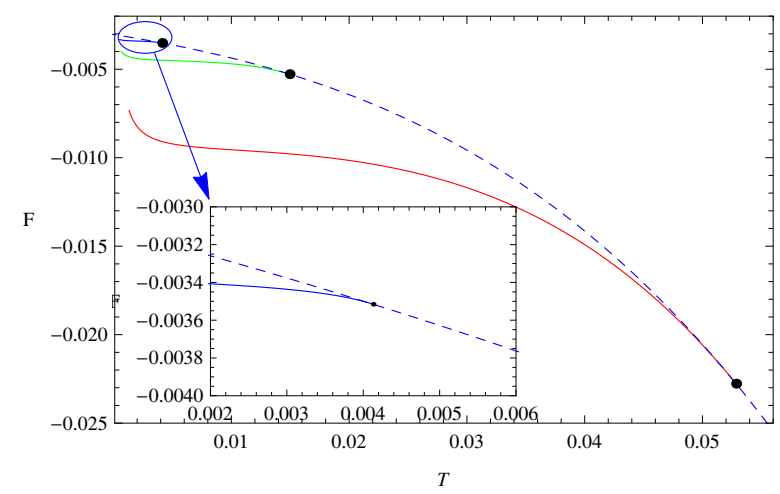

Figure 8. (Color online) The picture is for the behaviors of free energy with respect to the temperature with $\gamma=0.1, m^{2}=-2$ and $\zeta=0$. The curves from bottom to top correspond to the first (red), the second (green) and the third (blue) superconducting states. We have choose the chemical potential $\mu=1$ in both states. The blue dashed line shows the free energy of the pure AdS black hole. The black solid points correspond to the critical phase transition point between normal and superconductor phase.

canonical free energy. The solution in (a) of figure 1, figure 2 and figure 4 corresponding to the bottom red line in figure 8 has the lowest free energy and is thus the stable phase, whereas the solutions with higher free energy are unstable.

\section{Conclusions}

We investigated a general class of holographic superconductors via Stückelberg mechanism in the background of AdS black hole. We obtained richer structure in the metal/superconductor phase transitions. We observed that the model parameter coupled with the scalar mass and backreaction can determine the order of phase transitions. We found two threshold values $\tilde{\zeta}$ and $\bar{\zeta}$ for each pairs of scalar mass $m^{2}$ and backreaction parameter $\gamma$. When the model parameter satisfying $\zeta \leqslant \tilde{\zeta}$, there is only the second order phase transition at $T_{c}$. If $\tilde{\zeta}<\zeta<\bar{\zeta}$, the phase transition at $T_{c}$ is still the second order. However, an additional first order phase transition appears in the superconducting phase. When $\zeta \geqslant \bar{\zeta}$, it is the typical first order phase transition at the phase transition point $T_{c}$. We also tried to disclose the properties of the phase transitions by analyzing the entanglement entropy of the metal/suoerconductor system. We argued that the entanglement entropy serves as a good probe to the order of the phase transitions and the jump of the entanglement entropy would be a quite general feature for the first order phase transition in the AdS black hole background. In addition, we examined effects of the scalar mass and backreaction on the scalar condensation. We found that the less negative mass and stronger backreaction make all types of first order phase transitions easier to happen. Furthermore, we arrived at a relation $\bar{\zeta} \approx 2 \widetilde{\zeta}$ between the threshold parameters. With the shooting method, we obtained various superconductor solutions corresponding to different energy states. At last, we disclosed the stability of various energy states with the behaviors of the free energy. We 
concluded that the usually studied holographic superconductor solutions corresponding to the lowest energy state is stable, whereas the higher energy state is unstable.

\section{Acknowledgments}

We would like to thank Professor Bin Wang for his helpful discussions and suggestions on this topic. We also want to thank the anonymous referee for the constructive suggestions to improve the manuscript. This work was supported in part by the National Natural Science Foundation of China under Grant No. 11305097, the education department of Shaanxi province of China under Grant No. 2013JK0616 and the Foundation of Shaaxi University of Technology of China under Grant No. SLGQD13-23. This work was also supported by the China postdoctoral Science Foundation under Grant No. 2013M531163.

Open Access. This article is distributed under the terms of the Creative Commons Attribution License (CC-BY 4.0), which permits any use, distribution and reproduction in any medium, provided the original author(s) and source are credited.

\section{References}

[1] J.M. Maldacena, The large- $N$ limit of superconformal field theories and supergravity, Int. J. Theor. Phys. 38 (1999) 1113 [hep-th/9711200] [INSPIRE].

[2] S.S. Gubser, I.R. Klebanov and A.M. Polyakov, Gauge theory correlators from noncritical string theory, Phys. Lett. B 428 (1998) 105 [hep-th/9802109] [INSPIRE].

[3] E. Witten, Anti-de Sitter space and holography, Adv. Theor. Math. Phys. 2 (1998) 253 [hep-th/9802150] [INSPIRE].

[4] S.A. Hartnoll, Lectures on holographic methods for condensed matter physics, Class. Quant. Grav. 26 (2009) 224002 [arXiv:0903.3246] [INSPIRE].

[5] C.P. Herzog, Lectures on holographic superfluidity and superconductivity, J. Phys. A 42 (2009) 343001 [arXiv:0904.1975] [inSPIRE].

[6] G.T. Horowitz, Introduction to holographic superconductors, Lect. Notes Phys. 828 (2011) 313 [arXiv:1002.1722] [INSPIRE].

[7] G.T. Horowitz and M.M. Roberts, Holographic superconductors with various condensates, Phys. Rev. D 78 (2008) 126008 [arXiv:0810.1077] [inSPIRE].

[8] E. Nakano and W.-Y. Wen, Critical magnetic field in a holographic superconductor, Phys. Rev. D 78 (2008) 046004 [arXiv: 0804.3180] [INSPIRE].

[9] G. Koutsoumbas, E. Papantonopoulos and G. Siopsis, Exact gravity dual of a gapless superconductor, JHEP 07 (2009) 026 [arXiv:0902.0733] [INSPIRE].

[10] J. Sonner, A rotating holographic superconductor, Phys. Rev. D 80 (2009) 084031 [arXiv: 0903.0627] [INSPIRE].

[11] S.S. Gubser, C.P. Herzog, S.S. Pufu and T. Tesileanu, Superconductors from superstrings, Phys. Rev. Lett. 103 (2009) 141601 [arXiv:0907.3510] [INSPIRE].

[12] S.A. Hartnoll, C.P. Herzog and G.T. Horowitz, Holographic superconductors, JHEP 12 (2008) 015 [arXiv:0810.1563] [INSPIRE]. 
[13] Y. Liu, Q. Pan and B. Wang, Holographic superconductor developed in BTZ black hole background with backreactions, Phys. Lett. B 702 (2011) 94 [arXiv:1106.4353] [INSPIRE].

[14] Y. Liu, Q. Pan and B. Wang, Holographic superconductor developed in BTZ black hole background with backreactions, Phys. Lett. B 702 (2011) 94 [arXiv:1106.4353] [INSPIRE].

[15] J.P. Gauntlett, J. Sonner and T. Wiseman, Holographic superconductivity in M-theory, Phys. Rev. Lett. 103 (2009) 151601 [arXiv:0907.3796] [inSPIRE].

[16] J. Jing and S. Chen, Holographic superconductors in the Born-Infeld electrodynamics, Phys. Lett. B 686 (2010) 68 [arXiv: 1001.4227] [INSPIRE].

[17] C.P. Herzog, An analytic holographic superconductor, Phys. Rev. D 81 (2010) 126009 [arXiv: 1003.3278] [INSPIRE].

[18] K. Maeda, M. Natsuume and T. Okamura, Universality class of holographic superconductors, Phys. Rev. D 79 (2009) 126004 [arXiv:0904.1914] [InSPIRE].

[19] R. Gregory, S. Kanno and J. Soda, Holographic superconductors with higher curvature corrections, JHEP 10 (2009) 010 [arXiv:0907.3203] [INSPIRE].

[20] Q. Pan, B. Wang, E. Papantonopoulos, J. Oliveira and A.B. Pavan, Holographic superconductors with various condensates in Einstein-Gauss-Bonnet gravity, Phys. Rev. D 81 (2010) 106007 [arXiv:0912.2475] [INSPIRE].

[21] X.-H. Ge, B. Wang, S.-F. Wu and G.-H. Yang, Analytical study on holographic superconductors in external magnetic field, JHEP 08 (2010) 108 [arXiv:1002.4901] [INSPIRE].

[22] Y. Brihaye and B. Hartmann, Holographic superconductors in $3+1$ dimensions away from the probe limit, Phys. Rev. D 81 (2010) 126008 [arXiv: 1003.5130] [INSPIRE].

[23] Y. Brihaye and B. Hartmann, Holographic superfluid/fluid/insulator phase transitions in $2+1$ dimensions, Phys. Rev. D 83 (2011) 126008 [arXiv:1101.5708] [InSPIRE].

[24] S.S. Gubser, Breaking an Abelian gauge symmetry near a black hole horizon, Phys. Rev. D 78 (2008) 065034 [arXiv:0801.2977] [INSPIRE].

[25] S.A. Hartnoll, C.P. Herzog and G.T. Horowitz, Building a holographic superconductor, Phys. Rev. Lett. 101 (2008) 031601 [arXiv:0803.3295] [INSPIRE].

[26] C.P. Herzog, P.K. Kovtun and D.T. Son, Holographic model of superfluidity, Phys. Rev. D 79 (2009) 066002 [arXiv:0809.4870] [INSPIRE].

[27] G.T. Horowitz and B. Way, Complete phase diagrams for a holographic superconductor/insulator system, JHEP 11 (2010) 011 [arXiv:1007.3714] [INSPIRE].

[28] S. Franco, A. Garcia-Garcia and D. Rodriguez-Gomez, A general class of holographic superconductors, JHEP 04 (2010) 092 [arXiv: 0906.1214] [INSPIRE].

[29] S. Franco, A.M. Garcia-Garcia and D. Rodriguez-Gomez, A holographic approach to phase transitions, Phys. Rev. D 81 (2010) 041901 [arXiv:0911.1354] [INSPIRE].

[30] Q. Pan and B. Wang, General holographic superconductor models with Gauss-Bonnet corrections, Phys. Lett. B 693 (2010) 159 [arXiv:1005.4743] [INSPIRE].

[31] Y. Peng and Q.Y. Pan, Stückelberg holographic superconductor models with backreactions, Commun. Theor. Phys. 59 (2013) 110. 
[32] Y. Peng, Q. Pan and B. Wang, Various types of phase transitions in the AdS soliton background, Phys. Lett. B 699 (2011) 383 [arXiv:1104.2478] [INSPIRE].

[33] S. Ryu and T. Takayanagi, Holographic derivation of entanglement entropy from AdS/CFT, Phys. Rev. Lett. 96 (2006) 181602 [hep-th/0603001] [INSPIRE].

[34] S. Ryu and T. Takayanagi, Aspects of holographic entanglement entropy, JHEP 08 (2006) 045 [hep-th/0605073] [INSPIRE].

[35] T. Nishioka and T. Takayanagi, AdS bubbles, entropy and closed string tachyons, JHEP 01 (2007) 090 [hep-th/0611035] [INSPIRE].

[36] I.R. Klebanov, D. Kutasov and A. Murugan, Entanglement as a probe of confinement, Nucl. Phys. B 796 (2008) 274 [arXiv:0709.2140] [INSPIRE].

[37] A. Pakman and A. Parnachev, Topological entanglement entropy and holography, JHEP 07 (2008) 097 [arXiv: 0805.1891] [INSPIRE].

[38] T. Nishioka, S. Ryu and T. Takayanagi, Holographic entanglement entropy: an overview, J. Phys. A 42 (2009) 504008 [arXiv:0905.0932] [INSPIRE].

[39] L.-Y. Hung, R.C. Myers and M. Smolkin, On holographic entanglement entropy and higher curvature gravity, JHEP 04 (2011) 025 [arXiv:1101.5813] [INSPIRE].

[40] J. de Boer, M. Kulaxizi and A. Parnachev, Holographic entanglement entropy in Lovelock gravities, JHEP 07 (2011) 109 [arXiv:1101.5781] [INSPIRE].

[41] N. Ogawa and T. Takayanagi, Higher derivative corrections to holographic entanglement entropy for AdS solitons, JHEP 10 (2011) 147 [arXiv:1107.4363] [INSPIRE].

[42] T. Albash and C.V. Johnson, Holographic entanglement entropy and renormalization group flow, JHEP 02 (2012) 095 [arXiv: 1110.1074] [INSPIRE].

[43] R.C. Myers and A. Singh, Comments on holographic entanglement entropy and RG flows, JHEP 04 (2012) 122 [arXiv: 1202.2068] [INSPIRE].

[44] X.-M. Kuang, E. Papantonopoulos and B. Wang, Entanglement entropy as a probe of the proximity effect in holographic superconductors, JHEP 05 (2014) 130 [arXiv:1401.5720] [INSPIRE].

[45] W. Yao and J. Jing, Holographic entanglement entropy in metal/superconductor phase transition with Born-Infeld electrodynamics, Nucl. Phys. B 889 (2014) 109 [arXiv: 1408.1171] [INSPIRE].

[46] T. Albash and C.V. Johnson, Holographic studies of entanglement entropy in superconductors, JHEP 05 (2012) 079 [arXiv:1202.2605] [INSPIRE].

[47] R.-G. Cai, S. He, L. Li and Y.-L. Zhang, Holographic entanglement entropy on P-wave superconductor phase transition, JHEP 07 (2012) 027 [arXiv: 1204.5962] [INSPIRE].

[48] L.-F. Li, R.-G. Cai, L. Li and C. Shen, Entanglement entropy in a holographic P-wave superconductor model, arXiv:1310.6239 [INSPIRE].

[49] Y. Peng and Q. Pan, Holographic entanglement entropy in general holographic superconductor models, JHEP 06 (2014) 011 [arXiv: 1404.1659] [INSPIRE].

[50] R.-G. Cai, S. He, L. Li and L.-F. Li, Entanglement entropy and Wilson loop in Stückelberg holographic insulator/superconductor model, JHEP 10 (2012) 107 [arXiv:1209.1019] [INSPIRE]. 
[51] R.A. Konoplya and A. Zhidenko, Holographic conductivity of zero temperature superconductors, Phys. Lett. B 686 (2010) 199 [arXiv:0909.2138] [INSPIRE].

[52] G.T. Horowitz, Introduction to holographic superconductors, Lect. Notes Phys. 828 (2011) 313 [arXiv:1002.1722] [INSPIRE].

[53] R.-G. Cai, X. He, H.-F. Li and H.-Q. Zhang, Phase transitions in AdS soliton spacetime through marginally stable modes, Phys. Rev. D 84 (2011) 046001 [arXiv:1105.5000] [INSPIRE].

[54] T. Nishioka, S. Ryu and T. Takayanagi, Holographic superconductor/insulator transition at zero temperature, JHEP 03 (2010) 131 [arXiv:0911.0962] [INSPIRE].

[55] P. Breitenlohner and D.Z. Freedman, Positive energy in anti-de Sitter backgrounds and gauged extended supergravity, Phys. Lett. B 115 (1982) 197 [INSPIRE]. 